\title{
Extracts from Salvia-Nelumbinis naturalis alleviate hepatosteatosis via improving hepatic insulin sensitivity
}

\author{
Li Zhang ${ }^{1}$, Jiaoya Xu' ${ }^{1}$, Haiyan Song ${ }^{1}$, Zemin Yao $^{2}$ and Guang $\mathrm{Ji}^{1,3^{*}}$
}

\begin{abstract}
Background: Salvia-Nelumbinis naturalis (SNN), initially called Jiangzhi Granula as a formulae of Chinese medicinal decoction, has been used clinically to treat non-alcoholic fatty liver disease (NAFLD) and related syndromes. The mechanism of SNN action is unknown.

Methods: HepG2 cells were cultured in lipid-rich media supplemented with chemical components of SNN. Male Wistar rats ( 6 weeks of age) were fed a high calorie diet (15\% fat, 15\% sucrose, and 2\% cholesterol) for eight weeks, and then treated with SNN for four weeks. Body and liver weight, lipids profiles, insulin and glucose levels, glucose and insulin tolerance were evaluated, the mRNA and protein expression of insulin receptor (InsR), insulin receptor substrate (IRS) 1/2, protein kinase B (PKB/Akt), protein expression of suppressor of cytokine signaling 3 (SOCS3), protein kinase $C$ epsilon (PKC $\varepsilon$ ) in liver tissue were analysed.

Results: Treatment with SNN components in lipid-laden HepG2 cells decreased lipid accumulation. Rats fed with a HC diet developed hepatosteatosis and accompanied hyperglycemia, hyperinsulinemia, hyperleptinemia, and diabetic dyslipidemia. Prolonged HC diet feeding resulted in parabolic response in plasma triglyceride (TG) concentrations, indicative of compromised hepatic production of TG-rich lipoproteins. HC diet feeding also resulted in impaired insulin sensitivity and hepatic insulin signalling. Administration of SNN extracts alleviated hepatosteatosis and conferred to a normolipoproteinemia profile in the HC diet-fed rats. The efficacy of SNN extract in improving liver function and insulin sensitivity was comparable to that of simvastatin or pioglitazone. The improved insulin signaling by SNN treatment was associated with increased IRS and Akt phosphorylation and decreased SOCS3 expression. However, SNN failed to inhibit the PKC $\varepsilon$ expression in the liver.
\end{abstract}

Conclusions: SNN is effective in reducing lipid accumulation in HepG2 cells and attenuating hepatosteatosis in HC diet-fed rats. Reduced hepatic lipid content in the rat liver was associated with improved insulin signalling.

Keywords: Hepatosteatosis, Insulin resistance, Salvia-Nelumbinis naturalis

\section{Background}

Hepatosteatosis, i.e. excess accumulation of triglyceride (TG) in the liver, is the defining feature of non-alcoholic fatty liver disease (NAFLD). As the epidemic of obesity has increased worldwide, NAFLD become a critical public health issue because of its high prevalence, potential progression to severe liver disease, and association with

\footnotetext{
*Correspondence: jiliver@vip.sina.com

'Institute of Digestive Disease, Longhua Hospital, Shanghai University of

Traditional Chinese Medicine, Shanghai, China

${ }^{3}$ E-Institute of Shanghai Municipal Education Commission, Shanghai

University of Traditional Chinese Medicine, Shanghai, China

Full list of author information is available at the end of the article
}

serious metabolic abnormalities including type 2 diabetes mellitus, metabolic syndrome, and coronary heart disease [1]. Previously considered as an abnormality confined to adult populations, NAFLD, is now an alarming health issue among obese children [2]. Hence, finding an effective NAFLD therapy is necessary to alleviate the health burden caused by this disease.

Insulin resistance is intimately associated with the pathogenesis of NAFLD, as evidenced by patho-physiologic studies [3-5]. Insulin binds to its receptor, which results in the tyrosine phosphorylation of insulin receptor substrate (IRS)-1and IRS-2, and subsequently activates downstream

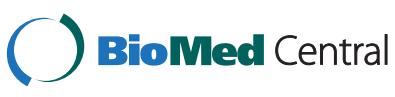

(c) 2014 Zhang et al.; licensee BioMed Central Ltd. This is an Open Access article distributed under the terms of the Creative Commons Attribution License (http://creativecommons.org/licenses/by/4.0), which permits unrestricted use, distribution, and reproduction in any medium, provided the original work is properly credited. The Creative Commons Public Domain Dedication waiver (http://creativecommons.org/publicdomain/zero/1.0/) applies to the data made available in this article unless otherwise stated. 
molecules such as phosphoinositide 3-kinase, protein kinase $\mathrm{B}$ (PKB/Akt), etc. [6]. Impaired insulin signalling, especially the paradox of selective hepatic insulin resistance of failing to suppress glucose production but preserved insulin sensitivity in lipid synthesis aggravates insulin resistance and contributes to the development of NAFLD, while regimens that reverse insulin resistance can ameliorate steatosis [7]. Although numerous studies have focused on NAFLD, suggested treatments (thiazolidinediones, statins) do not always produce satisfied results. Calorie restriction and exercise can treat simple or moderate NAFLD effectively, but target drugs are needed when the disease becomes progressive and severe.

For centuries, natural herbal products derived from systemic Traditional Chinese Medicine (TCM) theory and practice have been used to treat nearly all kinds of ailments in China. Formulae, the form of prescription in TCM, typically consists of several medicinal herbs, of which one represents a principal component, and others serve as an adjuvant in assisting the effects or facilitating the delivery of the principal component. Entirely based on TCM theory and NAFLD pathology, Salvia-Nelumbinis naturalis (SNN) formulae (initially called Jiangzhi Granula) was designed, in which Salviaeas being the principal element and Nelumbinis, Rhizoma Polygoni Cuspidati, Herba Artemisiae Scopariae the ancillary components. We have shown that administration of SNN formulae, together with behavioral intervention, to patients with NAFLD for 24 weeks gave rise to a higher remission rate as compared to behavioral intervention alone $(76.56 \%$ versus 52.54\%) [8]. In addition, with all the cases observed, SNN did not demonstrate any adverse effect during the whole process [8]. These promising clinical observations of the SNN formulae in ameliorating NAFLD have prompted us to define mechanisms responsible for SNN action. An UPLC-MS method has been established to determine the main active components of SNN [9], thus ensuring standardized application in clinical and preclinical studies.

High calorie $(\mathrm{HC})$ diet is a method that is extensively used to induce hepatic steatosis and insulin resistance in rats and mice [10-12], which resemble characteristics of NAFLD in human. In this study, a rat model of HC-induced NAFLD was used to examine the influence of the extract of SNN on NAFLD and its potential role in improving insulin resistance.

\section{Methods}

\section{Materials}

HepG2 cells were purchased from the American Type Culture Collection (ATCC) bioresource center (Manassas, VA, USA). Dulbecco's modified Eagle's medium (DMEM), fetal bovine serum (FBS), and penicillin and streptomycin were from Biowest (Nuaillé, France). The main chemical components of the SNN extract protopanaxadiol, tanshinone IIA, gypenoside, Salvianic acid A sodium, and Salvianolic acid B were obtained from Shanghai Winherb Medical Sci \& Tech Development Co. Ltd. (Shanghai, China). Pioglitazone were purchased from Sigma (St. Louis, MO, USA). All other reagents were of analytical grade.

\section{Cell culture}

HepG2 were cultured in DMEM supplemented with $10 \%$ heat-inactivated fetal FBS, penicillin at $100 \mathrm{U} / \mathrm{mL}$, and streptomycin at $100 \mu \mathrm{g} / \mathrm{mL}$ at $37^{\circ} \mathrm{C}$ in a $5 \% \mathrm{CO} 2$ atmosphere. The experiments, started when the cells grew to $80-90 \%$ confluence. Cellular steatosis was induced as previously described [13], in brief, HepG2 cells were seeded on 6-well plates $1 \times 10^{5}$ cells/well, stimulated by a mixture of $1 \mathrm{mmol} / \mathrm{L}$ FFA (the ratio of oleate to palmitate is 2:1) in DMEM containing 1\% BSA for $24 \mathrm{~h}$. The steatotic cells were then treated with the chemical components (at 5, 50, and $500 \mu \mathrm{mol} / \mathrm{L}$ concentration for each component) for another $24 \mathrm{~h}$. The cells were stained with Oil-Red $\mathrm{O}$ and imaged.

\section{Preparation of extract of Salvia-Nelumbinisnaturalis}

The Salvia-Nelumbinisnaturalis(SNN) formula composed of Salviae (Danshen in China) as the principal element and Nelumbinis (Heye in China), Rhizoma Polygoni Cuspidati (Huzhang in China), Herba Artemisiae Scopariae (Yinchen in China) as ancillary components. The plant specimens were under a micro plant grinding machine, triturated and blended to powder, and the powder was then mixed with water/methanol $(5: 95, \mathrm{~V} / \mathrm{V})$ by sonication (42 kHz, $30 \mathrm{~min}$ ) using an ultrasonic processor as described previously [9]. The main chemical components of the SNN extract has been determined by UPLC-MS system in previous publications [9].

\section{Animals and study design}

Male Wistar rats were obtained from Slac Animal Laboratories (Shanghai, China), and housed under a standard 12-h light-dark cycle (lights on at 7:00 AM) with access to food and water ad libitum. After approximately 1-week acclimation, rats were placed on either standard chow or HC (15\% fat, $15 \%$ sucrose and $2 \%$ cholesterol) diet for up to 12 weeks. During the last 4-week dieting, rats on $\mathrm{HC}$ diet were divided into four groups - one group continued on $\mathrm{HC}$ diet and the remainder three groups were administered (through oral gavage, daily) with pioglitazone $(10 \mathrm{mg} / \mathrm{kg}$ body weight), Simvastatin (4 mg/kg), and SNN extract $(600 \mathrm{mg} / \mathrm{kg})$, respectively. Oral gavage of saline was applied to control rats. The experimental protocols were approved by Shanghai University of Traditional Chinese Medicine. Rats were 
housed in a pathogen free environment, and body weight and 24-h food intake were measured weekly.

\section{Oral glucose tolerance test and insulin tolerance test}

Oral glucose tolerance test (OGTT) and insulin tolerance test (ITT)were performed with rats at the end of dieting using previously described methods [14]. Briefly, rats were fasted for $6 \mathrm{~h}$ after the start of the light cycle, then orally administered with glucose $(1.5 \mathrm{~g} / \mathrm{kg}$ body weight, for OGTT) or intraperitoneally administrtered with insulin $(0.75 \mathrm{u} / \mathrm{kg}$ body weight, for ITT), tail-vein blood samples were collected at baseline and at indicated time intervals $(15,30,60,90$, and $120 \mathrm{~min})$ after glucose/insulin administration. Blood glucose levels were determined with a diabetes monitoring strip (Lifescan One Touch, IN). The curve was drawn and the area under the curve (AUC) was calculated.

\section{Serum biochemical analysis}

After fasting for $12 \mathrm{~h}$, rats were anaesthetized with sodium pentobarbital $(100 \mathrm{mg} / \mathrm{kg})$ and sacrificed, and blood was collected from aorta abdominalis. Serum TG, total cholesterol (TC), high density lipid-cholesterol (HDL-c), low density lipid-cholesterol (LDL-c), free fatty acids (FFA), fasting blood glucose (FBG), almandine aminotransferase (ALT), and aspartate aminotransferase (AST) were analyzed using the Hitachi full-automatic system. Fasting insulin (FIN) was measured using the standard radio-immunity kits (Puerweiye Bioengineering Institute, Beijing, China).

\section{Hepatic histology assessment}

Liver sections were stained with Hematoxylin and eosin (HE) and Oil-Red O (Sigma, St. Louis, MO), the procedures were performed according to previously describe methods [15]. Briefly, the liver tissues were fixed in 10\% neutral buffered formalin for $24 \mathrm{~h}$, dehydrated and embedded in paraffin, the sections were cut, deparaffinized and stained with HE. Snap frozen tissues were placed in optimal cutting temperature compound and then sectioned and stained with Oil-Red O.

\section{Hepatic lipid and glycogen content}

Liver TG was estimated as described previously [16]. Briefly, liver tissue $(200 \mathrm{mg})$ was homogenized in $3 \mathrm{ml}$ of ethanol-acetone (1:1) mixture. The homogenate was extracted over night at $4^{\circ} \mathrm{C}$, and centrifuged for $15 \mathrm{~min}$ at 3,000 rpm at $4^{\circ} \mathrm{C}$. The organic layer was removed, TG and cholesterol were measured using commercial kits (Kangtai Bioengineering Institute, Beijing, China). A standard kit (Jianchen tech, Nanjing, China) was used to determine the hepatic glycogen concentration.

\section{RNA analysis}

Total RNA was extracted from liver and hypothalamic tissues by using a TRIzol Reagent (Invitrogen Corp., Carlsbad, CA) following the manufacturer's instructions. A reverse-transcription system (Promega Corp., Madison, WI) was then used to transcribe the RNA. Semi-quantitative reverse transcription polymerase chain reaction method was performed using Mastercycler (Eppendorf, Inc., Germany). The primer sequences are shown in Table 1. The mRNA levels of all genes were normalized using $\beta$-actin as an internal control.

\section{Tissue sampling and western blot analyses}

Frozen liver and hypothalamic tissues were homogenized in Tissue Protein Extraction Reagent (Pierce Biotechnology, Inc., Rockford, USA), with the addition of protease inhibitor (Roche, Nutley, USA) and phosphatase inhibitor cocktail (Roche, Nutley, USA). Protein concentrations were determined using the bicinchoninic assay reagents and the micro-bicinchoninic assay method (Pierce Biotechnology, Inc. Rockford, USA). For Western blot analysis, $100 \mu \mathrm{g}$ of protein were fractionated by $8-12 \%$ SDS-PAGE, transferred onto a PVDF membrane (Bio-Rad, Hercules, CA), Membranes were blocked with 5\% skim milk in Tris-buffered saline and probed with target primary and secondary antibodies. Total and phosphorylated InsR $\left(\mathrm{Tyr}^{1131}\right)$, IRS1 $\left(\mathrm{Ser}^{302}\right)$, Akt $\left(\mathrm{Ser}^{473}\right)$ and SOCS3 antibodies were purchased from Cell Signaling Technology (Danvers, MA, USA), PKCe antibody was from Boster company (Wuhan, China). $\beta$-actin and secondary antibodies (from mouse or rabbit) conjugated to peroxidase were purchased from Santa Cruz Biotechnology. The targeted proteins were detected with ECL Detection Kit (Millipore, Billerica, USA), images were taken and qualified by Gel-Pro system (Tanon Technologies).For western blot analysis, the amount of protein loaded was confirmed by the Bradford method, and equal loading was verified by staining with Ponceau S reagent (Sigma Chemical Co.) and by determining the signal of $\beta$-actin.

\section{Statistical analysis}

For each outcome measure, a one-way analysis of variance was performed (SPSS 18.0) for each animal group studied $(\mathrm{n}=8)$. A significant main effect $(p<0.05$ or $p<0.01)$ was followed-up with Student-Newman-Kuel

Table 1 Sequences of the primers used for amplification of mRNA by PCR

\begin{tabular}{lll}
\hline Gene & Forward primer & Reverse primer \\
\hline InsR & TCTTCAGGGCAATGTCGTTC & GCTCCTATGCTCTGGTGTCA \\
IRS1 & TGCTGGTGGAAGAGGAGG & TGGACAAACGGAGTAGGG \\
IRS2 & GACCAAGTCGGTGAGTGC & GCCCGAACCTCAATAACA \\
B-actin & CTGGAAGGTGGACAGTGAG & GAGGGAAATCGTGCGTGAC \\
\hline
\end{tabular}




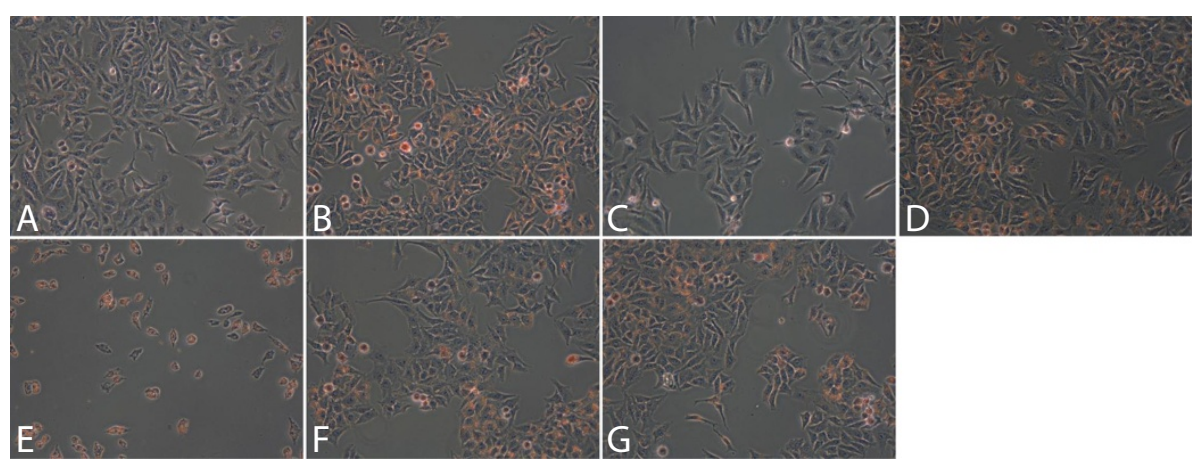

Figure 1 Effects of SNN chemical components on HepG2 cellular steatosis. HepG2 cells were stimulated by a mixture of 1 mmol/L FFA (the ratio of oleate to palmitate is 2:1) $24 \mathrm{~h}$. The steatotic cells were then treated with the chemical components for another $24 \mathrm{~h}$. The cells were

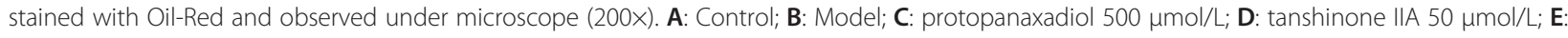
gypenoside $50 \mu \mathrm{mol} / \mathrm{L} ; \mathbf{F}$ : Salvianic acid A sodium $500 \mu \mathrm{mol} / \mathrm{L}$ G: Salvianolic acid B $50 \mu \mathrm{mol} / \mathrm{L}$.

post hoc comparisons. Values are presented as means \pm standard error (SE), and $p<0.05$ denotes a statistically significant difference.

\section{Results}

Effect of SNN components on steatosis in HepG2 cells The $24 \mathrm{~h}$ incubation HepG2 cells with FFA induced significant lipid accumulation in the cells compared to that of control cells (Figure 1A\&B). Treatment with different SNN components to the cell could reduce the lipid to different extent (Figure 1C-G). Notably, protopanaxadiol $(500 \mu \mathrm{mol} / \mathrm{L})$, Salvianic acid A sodium $(500 \mu \mathrm{mol} / \mathrm{L})$, and Salvianolic acid B $(50 \mu \mathrm{mol} / \mathrm{L})$ showed the obvious improvement of the steatosis, indicating the promising effect of SNN components on steatosis in HepG2 cells.

Parabolic response of serum lipids during hepatosteatosis progressing

Male Wistar rats placed on a $\mathrm{HC}$ diet exhibited the expected hypertriglyceridemia, hypercholesterolemia, hyperglycemia, hyperinsulinemia, hyperleptinemia, and increased body weight (Table 2) as compared to age matched controls. The serum FFA levels were increased by $30 \%$ at the end of 8-week of $\mathrm{HC}$ dieting (Table 3); however, the increased fasting FFA concentration upon prolonged $\mathrm{HC}$ dieting was not associated with increase in fasting serum TG. Rather, fasting serum TG exhibited gradual decline from 2-week of $\mathrm{HC}$ dieting (Figure 2A), even though the hepatic TG content remained high (Table 2). At the end of 12-week of HC dieting, the serum TG concentration was decreased to a level that was lower than that in chow diet-fed animals (chow diet: $1.62 \pm 0.80 \mathrm{mM}$, HC diet: $0.98 \pm 0.16 \mathrm{mM} ; P<0.01)$. Similarly, serum TC level also showed a trend of decline after the max at 1-week checkpoint, while hepatic cholesterol reached the plateau (Figure 2B). The decrease in plasma TG probably reflects the compromised ability of the liver to produce VLDL after prolonged HC dieting.

\section{Effect of SNN on liver steatosis}

The 8-week $\mathrm{HC}$ fed rats developed hepatomegaly; the mean liver weight and liver weight/body weight ratio

Table 2 Liver Parameters

\begin{tabular}{|c|c|c|c|c|c|c|c|}
\hline \multirow[t]{2}{*}{ Parameters } & \multicolumn{2}{|c|}{ 8-week dieting } & \multicolumn{5}{|c|}{ Post 4-week treatment } \\
\hline & Chow & $\mathrm{HC}$ & Chow & $\mathrm{HC}$ & $\mathrm{HC}+$ Pio & $\mathrm{HC}+\mathrm{SNN}$ & $\mathrm{HC}+\mathrm{Sim}$ \\
\hline Body weight (g) & $392.6 \pm 5.9$ & $433.3 \pm 6.1^{\text {tt }}$ & $436.0 \pm 6.5$ & $487.1 \pm 10.7^{* *}$ & $510.4 \pm 11.5$ & $477.3 \pm 9.5$ & $477.9 \pm 18.9$ \\
\hline Post 4-week body weight gain (g) & ND & ND & $43.38 \pm 3.82$ & $53.88 \pm 6.81^{*}$ & $78.73 \pm 8.24^{\#}$ & $45.00 \pm 5.96$ & $49.50 \pm 7.88$ \\
\hline Liver weight (g) & $9.9 \pm 0.07$ & $18.7 \pm 0.40^{\dagger t}$ & $14.05 \pm 0.39$ & $20.08 \pm 0.67^{* *}$ & $18.55 \pm 0.48$ & $19.78 \pm 0.70$ & $20.72 \pm 1.07$ \\
\hline Liver/Body weight ratio (\%) & $2.69 \pm 0.02$ & $4.31 \pm 0.03$ & $3.15 \pm 0.02$ & $4.12 \pm 0.17$ & $3.64 \pm 0.14$ & $4.14 \pm 0.22$ & $4.32 \pm 0.22$ \\
\hline Food intake (kal/d) & $73.5 \pm 3.1$ & $102.2 \pm 3.8^{t+}$ & $75.60 \pm 4.0$ & $110.24 \pm 5.2^{* *}$ & $112.36 \pm 3.8$ & $106.54 \pm 6.1$ & $104.88 \pm 5.6$ \\
\hline Liver TG (mg/g) & $20.2 \pm 0.6$ & $58.3 \pm 1.2^{\dagger+}$ & $24.1 \pm 0.6$ & $67.2 \pm 2.6^{* *}$ & $48.1 \pm 0.9^{\# \#}$ & $47.8 \pm 1.3^{\# \#}$ & $46.2 \pm 13^{\# \#}$ \\
\hline Liver glycogen (mg/g) & ND & ND & $46.7 \pm 4.57$ & $16.8 \pm 1.33^{* *}$ & $30.8 \pm 1.95^{\# \#}$ & $31.02 \pm 1.62^{\# \#}$ & ND \\
\hline ALT (u/l) & $51.6 \pm 2.8$ & $54.4 \pm 2.7$ & $56.04 \pm 8.91$ & $52.28 \pm 5.60$ & $62.51 \pm 8.68$ & $70.19 \pm 12.76$ & $66.4 \pm 8.75$ \\
\hline AST (u/l) & $125.5 \pm 12.9$ & $88.8 \pm 3.6$ & $110.1 \pm 10.79$ & $91.4 \pm 12.59$ & $90.2 \pm 15.35$ & $132.4 \pm 87.98$ & $89.7 \pm 8.70$ \\
\hline
\end{tabular}

Values are means $\pm \mathrm{SE}$ ( $\mathrm{n}=6$-8 per group). ${ }^{+\dagger} p<0.01$ relative to 8-weekunder chow diet, ${ }^{*} p<0.05,{ }^{* *} p<0.01$ relative to 12 -weekunder chow diet; ${ }^{\#} p<0.05$,

$\#$ \# $p<0.01$, versus 12-weekHC diet. TG, triglyceride; $A L T$, alanine transaminase; $A S T$, aspartate transaminase; $N D$, not determined. 
Table 3 Serum Parameters

\begin{tabular}{|c|c|c|c|c|c|c|c|}
\hline \multirow[t]{2}{*}{ Parameters } & \multicolumn{2}{|c|}{ 8-week dieting } & \multicolumn{5}{|c|}{ Post 4-week treatment } \\
\hline & Chow & $\mathrm{HC}$ & Chow & $\mathrm{HC}$ & $\mathrm{HC}+$ Pio & $\mathrm{HC}+\mathrm{SNN}$ & $\mathrm{HC}+\mathrm{Sim}$ \\
\hline $\mathrm{TG}(\mathrm{mM})$ & $1.12 \pm 0.08$ & $1.90 \pm 0.14^{t t}$ & $1.62 \pm 0.80$ & $0.98 \pm 0.16^{* *}$ & $0.88 \pm 0.23$ & $1.53 \pm 0.38^{\# \#}$ & $1.41 \pm 0.22^{\# \#}$ \\
\hline $\mathrm{TC}(\mathrm{mM})$ & $1.59 \pm 0.04$ & $2.95 \pm 0.13^{t t}$ & $1.93 \pm 0.18$ & $2.53 \pm 0.18^{* *}$ & $2.43 \pm 0.13$ & $2.44 \pm 0.14$ & $2.46 \pm 0.15$ \\
\hline $\mathrm{HDL}-\mathrm{c}(\mathrm{mM})$ & $1.02 \pm 0.01$ & $0.80 \pm 0.04^{t+}$ & $1.66 \pm 0.15$ & $0.95 \pm 0.09^{* *}$ & $1.12 \pm 0.12^{\#}$ & $0.99 \pm 0.06$ & $0.88 \pm 0.03$ \\
\hline LDL-c (mM) & $0.32 \pm 0.01$ & $1.60 \pm 0.08^{t t}$ & $0.25 \pm 0.07$ & $1.47 \pm 0.18^{* *}$ & $1.19 \pm 0.16^{\#}$ & $1.17 \pm 0.15^{\#}$ & $1.31 \pm 0.11$ \\
\hline FFA $(u / l)$ & $1792 \pm 81.6$ & $2414 \pm 145^{t+}$ & $1966 \pm 107$ & $2562 \pm 153^{* *}$ & $2162 \pm 136^{\#}$ & $2100 \pm 162^{\#}$ & $2476 \pm 174$ \\
\hline $\mathrm{FBG}(\mathrm{mM})$ & $5.0 \pm 0.11$ & $8.8 \pm 0.26^{t+}$ & $5.5 \pm 0.26$ & $9.8 \pm 0.26^{* *}$ & $9.6 \pm 0.33$ & $9.7 \pm 0.29$ & ND \\
\hline FIN (u/l) & $36.7 \pm 1.27$ & $194.5 \pm 25.47^{t \dagger}$ & $56.4 \pm 14.6$ & $226.3 \pm 19.7^{* *}$ & $208.0 \pm 19.4$ & $219.3 \pm 20.2$ & ND \\
\hline
\end{tabular}

Values are means $\pm \mathrm{SE}$ ( $\mathrm{n}=6$-8 per group). ${ }^{\mathrm{t+}} p<0.01$ relative to 8-weekunder chow $\operatorname{diet}^{* *} p<0.01$ relative to 12 -weekunder chow diet; ${ }^{\#} p<0.05,{ }^{\# \#} p<0.01$, versus12-weekHFHS diet.TG, triglyceride; $T C$, total cholesterol; $H D L-c$, high density lipoprotein cholesterol; $L D L-c$, low density lipoprotein cholesterol; $F F A$, free fatty acid; $F B G$, fasting blood glucose, FIN, fasting insulin; $N D$, not determined.

were 2- and 1.6-fold, respectively, higher than that in age matched rats fed chow diet (Table 2). Massive macro- vesicular steatosis were observed in liver sections in accordance with the increase of hepatic TG concentrations, indicating the development of fatty liver in HFHS fed rats. After 4 weeks of SNN treatment, however, hepatic steatosis was markedly reduced, despite the persistence of hepatomegaly (Figure 3). Hepatic TG concentrations in
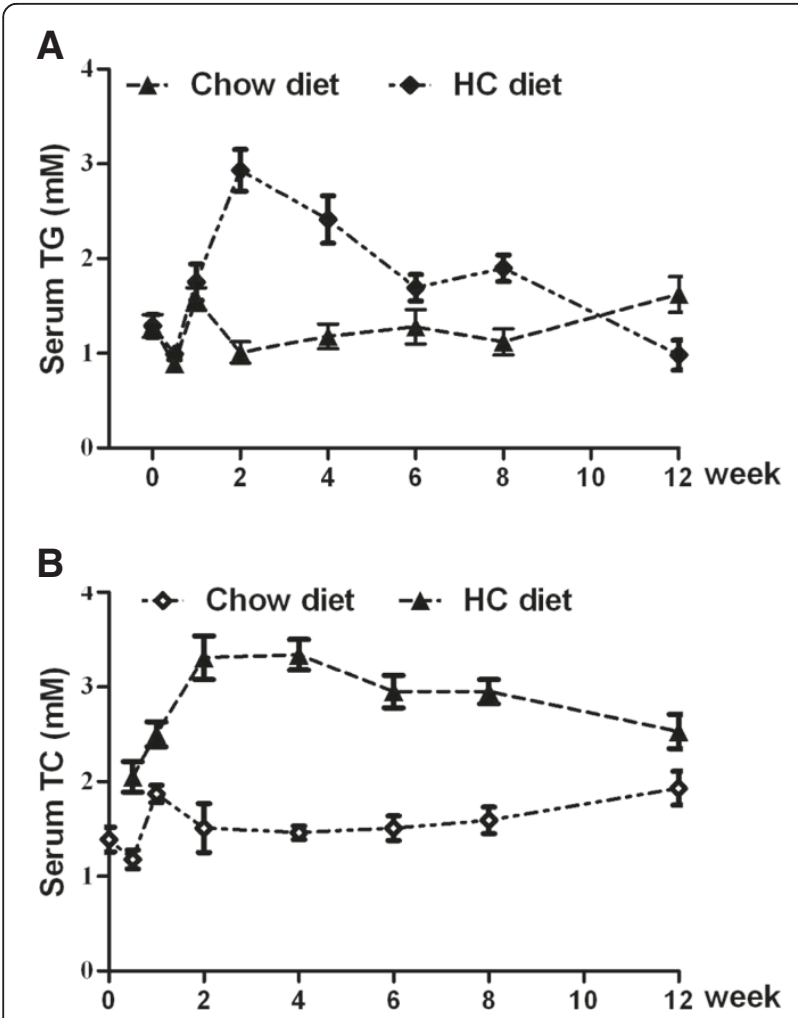

Figure 2 Parabolic decline of serum triglyceride level upon hepatosteatosis progress. Male Wistar rats ( 6 weeks of age) placed on a high-calorie $(\mathrm{HC})$ diet or normal chow diet, blood was collected at $0,2,4,6,8,10,12$ week, serum was separated and analysed. A:

The change of serum TG upon HC feeding; $\mathbf{B}$ : The change of serum TC upon HC feeding.
SNN-treated rats were decreased by approximately $30 \%$ as compared to that of non-treated control animals, and the effect of SNN treatment in reducing hepatic TG content was comparable to that of Simvastatin and Pioglitazone (Figure 3, Table 2).

\section{Effect of SNN on serum lipid profile}

Analysis of serum lipoproteins revealed that the hypercholesterolemia in rats fed the $\mathrm{HC}$ diet was associated with increased LDL-c and decreased HDL-c (Table 3), typical phenotypes of diabetic dyslipidemia. Rats fed with $\mathrm{HC}$ diet for 12 weeks also showed increased FFA levels in circulation (Table 3). The 4-week SNN treatment normalized the lipid profile to a certain extent, as manifested by decreased LDL-c and FFA (Table 3).

Increase hepatic fat content often correlates with increased TG secretion in the form of VLDL. However, decrease in serum TG is often observed as fatty liver progresses as a result of impaired hepatocellular function. The decrease in serum TG in HC diet-fed rats was reversed by 4-week SNN treatment (Table 3), suggesting restoration of normal hepatic TG secretion and hepatocellular function.

\section{Effect of SNN on insulin sensitivity}

$\mathrm{HC}$ diet-fed rats showed hyperinsulinemia and hyperglycemia, elevated HOMA-IR and impaired glucose tolerance compared with chow diet-fed controls (Table 2), indicative of insulin resistance. With 4-week SNN treatment, decrease in blood glucose levels was observed as compared with that in untreated $\mathrm{HC}$ rats at all time points (from 30 min through $2 \mathrm{~h}$ ) during the OGTT experiment (Figure 4A). Accordingly, decrease in OGTT-AUC values ensured in the SNN-treated rats (Figure 4B). As for ITT, there is no significant difference of the blood glucose between control group and $\mathrm{HC}$ diet group (Figure 4C), and so did the ITT-AUC values (Figure 4D). The 12-week $\mathrm{HC}$ feeding also resulted in a $60 \%$ decrease of hepatic glycogen content as compared to chow diet-fed rats, 


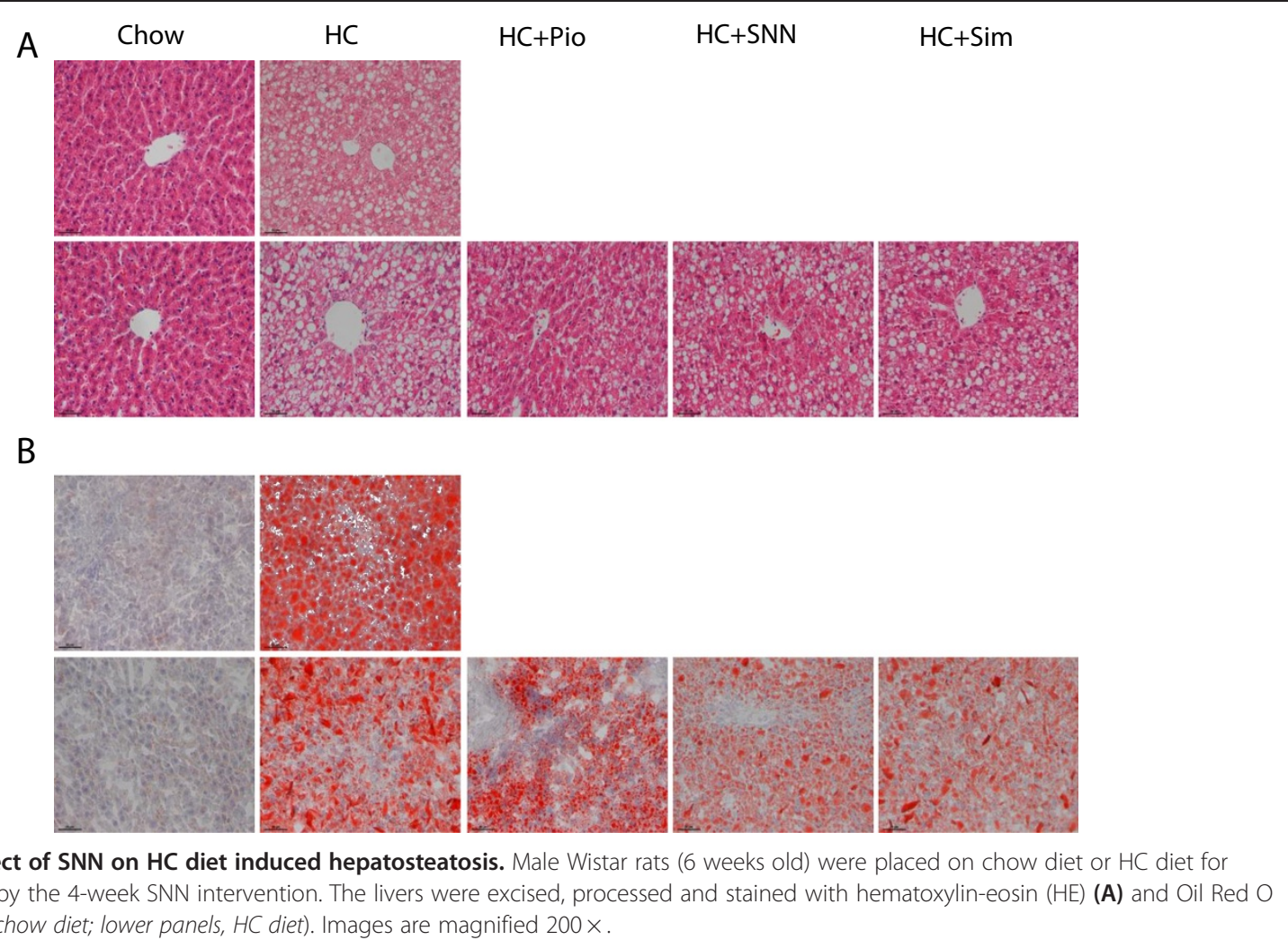

and SNN treatment partially normalized the hepatic glycogen content (Figure 4E), and the effect of SNN was comparable to that of pioglitazone.

In comparison to that in rats fed chow diet, the levels of InsR and IRS mRNA and IRS1 phosphorylation were decreased in rats fed $\mathrm{HC}$ diet (Figure 5). To demonstrate the effect of SNN in regulating insulin signalling transduction, we analysed molecular expression in insulin signalling pathway. With SNN supplement, the phosphorylated InsR and IRS2 levels were not significantly different from that of untreated rats, but IRS1 mRNA expression (Figure 5A) and phosphorylation protein (Figure 5B) dramatically increased, indicating the restoration of IRS1 activity. Akt is one of the downstream molecules of IRS in insulin pathway, which is critical in glucose homeostasis because it controls both gluconeogenesis and glycogen synthesis. The Akt phosphorylation in the 12-week HC rats were remarkably decreased, which may partially explain the $60 \%$ decrease of hepatic glycogen content compared with the chow diet animals. SNN increased the hepatic Akt phosphorylation (Figure 5A) and glycogen content (Figure 5B) with the 4-week treatment. These data together suggest strongly an improvement in hepatic insulin sensitivity with SNN treatment.

Effect of SNN on regulatory molecules of insulin pathway The abnormalities in lipid and glucose metabolism upon $\mathrm{HC}$ dieting were also associated with activation of regulatory signaling pathways. HC dieting induced increased expression of SOCS3, a negative regulator insulin signaling. SOCS3 has been shown interfering insulin signaling by competitively binding to the InsR, affecting IRS phosphorylation [17], or promoting ubiquitin-mediated IRS degradation [18]. Indeed, upon HC dieting, SOCS3 expression in the liver was up-regulated upon 12-week HC dieting, and with 4-week SNN treatment, SOCS3 expression was significantly decreased (Figure 6), indicating SNN has an inhibitory effect on SOCS3 expression, and the change was also consistent with IRS expression. In addition to SOCS3, the lipid-dependent kinases, PKC family has been reported to inhibit several components of the insulin signaling pathway, among the 10 isoforms, PKCE is the isoform most often implicated in association with hepatic insulin resistance [19]. Western blot analysis revealed that $\mathrm{PKC} \varepsilon$ was markedly increased along with the development of hepatic steatosis, however, the regulation of SNN on $\mathrm{PKC} \varepsilon$ was statistically no significant (Figure 6).

\section{Discussion}

Natural plant products provide a number of promising therapeutic agents. TCM uses a large variety of such herbal products and provides a rational prescription approach that targets the pattern of the syndrome, which is the essence of its therapeutic strategy. A lipid-lowering agent SNN was designed based on the characteristics of NAFLD and on 

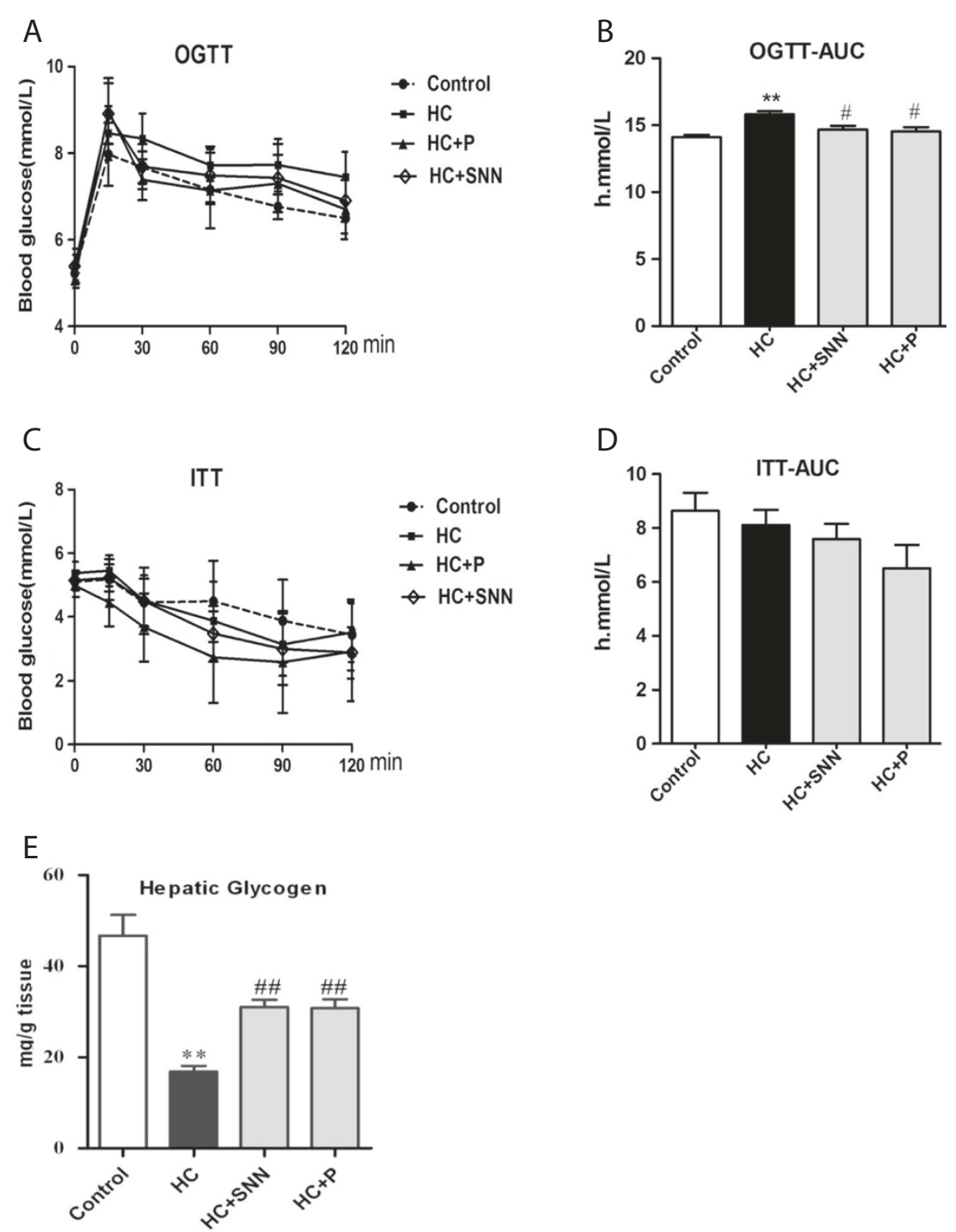

Figure 4 The effect of SNN on glucose and insulin tolerance and glycogen. Male Wistar rats ( 6 weeks old) were placed on chow diet or HC diet for 8 weeks followed by the 4-week SNN intervention. OGTT and ITT were conducted after $6 \mathrm{~h}$ fasting, glucose (1.5 g/kg body weight) was orally administered, or insulin $(0.75 \mathrm{u} / \mathrm{kg}$ body weight) was intraperitoneally injected, tail-vein blood samples were collected at baseline and at indicated time intervals $(15,30,60,90$, and $120 \mathrm{~min})$ after glucose administration. Blood glucose levels were determined with a diabetes monitoring strip. OGTT and ITT curves were drawn (A, C) and AUC were calculated (B, D). The livers were excised and glycogen content was detected $(\mathbf{E})$. Data (mean \pm SE) are presented, ${ }^{* *} P<0.01$ relative to control group, ${ }^{\#} P<0.05$, ${ }^{\# \#} P<0.01$ in comparison to $\mathrm{HC}$ group.

TCM theory. Clinical studies confirmed SNN as a therapeutic option for the development and progression of NAFLD [20]. The current study has demonstrated the SNN chemical components can reduce FFA-induced cellular steatosis. While HC diet-induced NAFLD rats exhibited dyslipidemia, hyperinsulinemia, and insulin resistance with concomitant hepatic steatosis, SNN reduced serum levels of LDL-c, and hepatic levels of TG with an attenuation of hepatic steatosis in $\mathrm{HC}$ diet-induced rats. These results together indicate that SNN is an effective drug for treating NAFLD.

Hepatic steatosis is the hallmark of NAFLD, and TG accumulation in hepatocytes is considered to be the major pathogenic trigger in the development of NAFLD [21]. In the current study, SNN components could improve FFA-induced steatosis in HepG2 cells, and animal studies showed that SNN could significantly attenuate the HC dietinduced hepatic steatosis and the lipid accumulation, both 

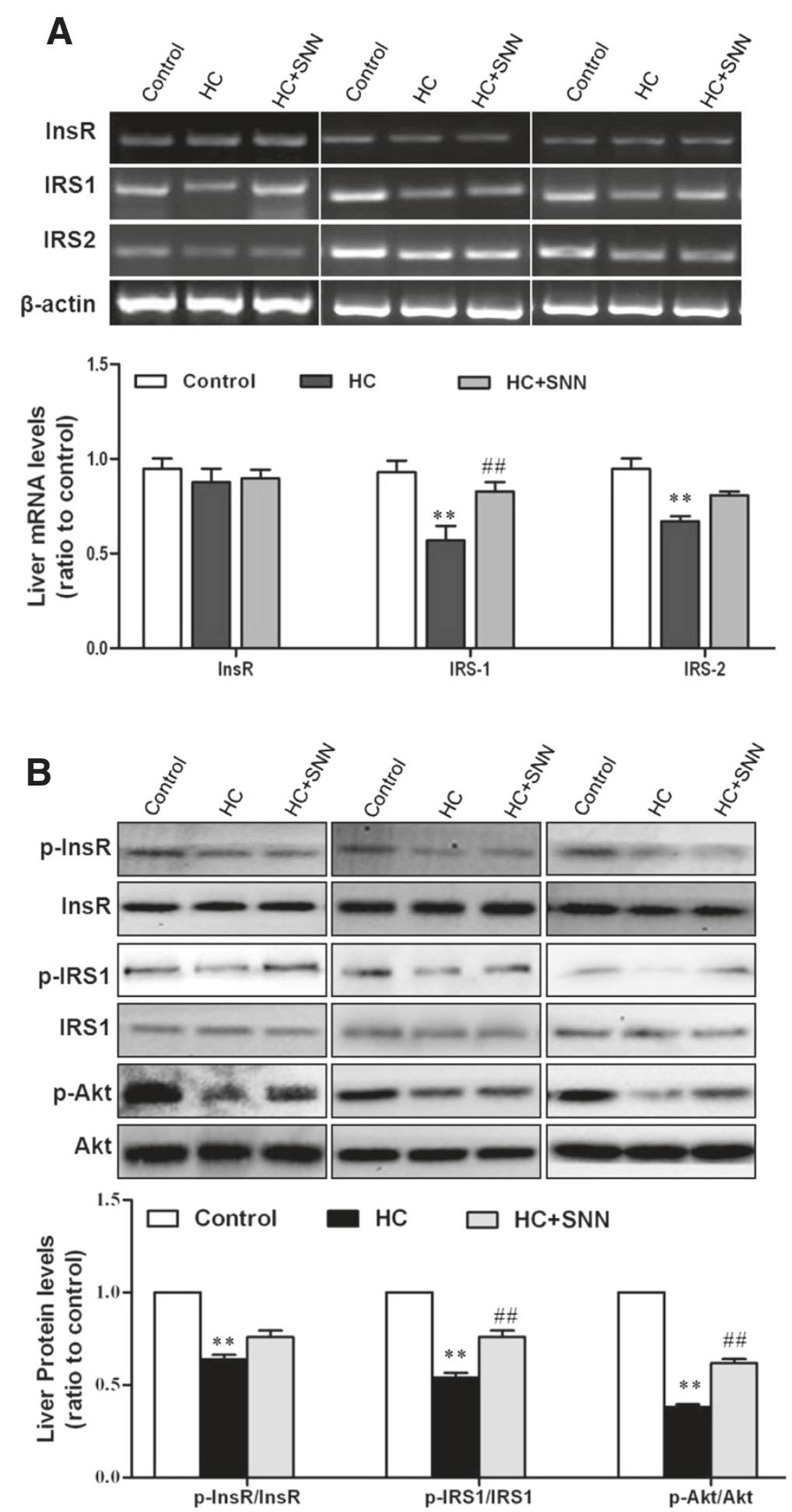

Figure 5 The effect of SNN on InsR, IRS1/2 and Akt expression in the liver. Male Wistar rats (6 weeks old) were placed on chow diet or HC diet for 8 weeks followed by the 4-week SNN intervention. Livers were collected, the mRNA expression of InsR and IRS1/2 was detected through PCR (A), and the protein expression of Total and phosphorylated InsR (Tyr $\left.{ }^{1131}\right)$, IRS1 (Ser $\left.{ }^{302}\right)$, Akt (Ser ${ }^{473}$ ) was analysed by Western blots (B). Data (mean \pm SE) are presented as relative expression levels compared to that of control group. ${ }^{* *} P<0.01$ relative to control group, ${ }^{\# \#} P<0.01$ in comparison to HC group. 


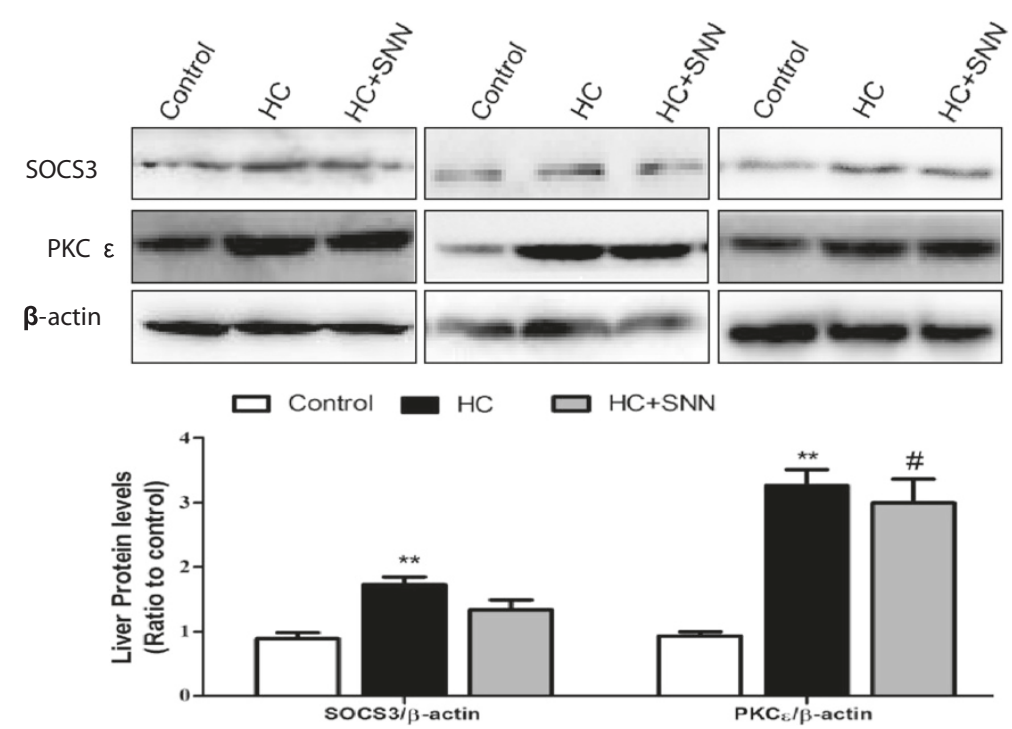

Figure 6 The effect of SNN on SOCS3 and PKCE expression in the liver. Male Wistar rats ( 6 weeks old) were placed on chow diet or HC diet for 8 weeks followed by the 4-week SNN intervention. Livers were collected, the protein expression of SOCS3 and PKC $\varepsilon$ was analysed by Western blots. Data (mean \pm SE) are presented as relative expression levels compared to that of control group. ${ }^{* *} P<0.01$ relative to control group, ${ }^{\#} P<0.05$ in comparison to $\mathrm{HC}$ group.

of which confirmed the therapeutic effect of SNN. Liver also overproduces several atherogenic factors under nutritional stress [22,23], in this study, the levels of and LDL-c were significantly decreased in SNN-treated rats, which partly confirms the protective nature of SNN against atherogenic risk.

NAFLD is associated with dyslipidemia, increased hepatic fat content often (but not always) correlates with increased hepatic TG secretion. The only route for TG export from hepatocytes is via the assembly of VLDL. However, as NAFLD progresses, the production of VLDL might decrease secondary to the impairment of hepatocellular function. Studies of seven patients with biopsy proven NASH showed decreased apolipoprotein B (apoB, the primary apolipoproteins of VLDL) production rate (by 50\%) as compared with obese or lean controls without NAFLD [24]. We have observed a parabolic response of serum TG level during diet-induced fatty liver progression, indicating the impairment of hepatocellular function. The mechanisms responsible for the parabolic change of serum TG is unclear. Nevertheless, 4-week SNN treatment results in an improved hepatocellular function, evidenced by the restoration of serum TG. Cell culture and animal studies showed that, although moderate FFA overload increases VLDL secretion, prolonged FFA exposure induced ER stress and resulted in decreased VLDL secretion [25]. Whether or not SNN treatment can reduce ER stress remains to be determined.

The two-hit and multi-hit hypotheses postulate that insulin resistance is the initial trigger of the pathogenesis of NAFLD [26,27]. Insulin sensitivity/resistance is usually tested on glucose metabolism. HOMA-IR is largely used in epidemiological studies because it only requires measuring FIN and FBG, and OGTT indices are frequently used in clinical practice $[28,29]$. Both tests are commonly used to measure whole-body and peripheral insulin resistance. The results of the experiment in this study showed an increase of HOMA-IR and OGTT indices, which signify insulin insensitivity in NAFLD rats. SNN markedly reduces OGTT indices, but fails to inhibit HOMA-IR increase, which suggests that SNN may not completely reverse whole-body insulin resistance involved in NAFLD.

Insulin has a strong influence on lipogenesis and glucose homeostasis as well [30,31]. The failure of hepatocytes to respond to insulin contributes to the development of glucose intolerance, whereas lipogenesis, which is positively regulated by insulin, remains sensitive to insulin and is driven excessively by the compensatory hyperinsulinemia [32]. Thus, the failure of the insulin system enhances hepatic glucose and lipid production [33]. Hepatic insulin resistance is strongly associated with NAFLD and is characterized by the impairment of glycogenesis with an increase of glucogenesis and glycogenolysis [34]. Glycogen synthesis represents a major pathway for glucose disposal after insulin stimulation, and increased hepatic glycogen synthesis and glycogen content are involved in enhanced insulin sensitivity [35]. Previous studies suggested that glycogen in the liver is important to maintain physical performance during prolonged exercise, and that glycogen synthesis is reduced in type 2 diabetes mellitus [36,37]. The present study demonstrated that SNN increases hepatic 
glycogen content, which is parallel to its improvement of insulin signalling transduction in NAFLD rats. The decrease in liver InsR and IRS expression is in accordance with the impairment of the hepatic insulin pathway and represents the therapeutic targets of insulin resistance [16]. This finding indicates that the improvement of hepatic insulin sensitivity contributes to the reduction of hepatic lipid accumulation in NAFLD rats. Therefore, hepatic insulin resistance is an important target for SNN therapy for HC diet-induced NAFLD.

Hepatic insulin resistance is also associated with activation of regulatory signaling pathways. Among the regulatory molecules, SOCS3 has been shown interfering insulin signaling by competitively binding to the InsR, affecting IRS phosphorylation [17], or promoting ubiquitin-mediated IRS degradation [18]. In addition to SOCS3, the isoforms of PKC family have wide-ranging roles in signal transduction, including the positive and negative modulation of insulin action, and they are dependent on diacylglycerol (DAG) for full activation [38]. PKCe is the isoform most often implicated in association with hepatic insulin resistance, $\mathrm{PKC} \varepsilon$ in liver may affect its ability to phosphorylate the substrates or InsR trafficking in hepatocytes [39]. In our study, both SOCS3 and PKCE were significantly increased with the development of NAFLD, which was consistent with the impairment of insulin signaling pathway, indicating these regulatory molecules may contribute to the insulin resistance. SNN showed improvement on SOCS3 expression, but not that of $\mathrm{PKC \varepsilon}$, the potential mechanisms need to be further explored, other isoforms of PKC family may also involved in the regulatory process.

Thiazolidinediones are insulin-sensitizing agents that improve insulin sensitivity and are effective for the treatment of specific metabolic defects. However, the use of thiazolidinediones for NAFLD treatment raises a number of safety concerns $[40,41]$. In this study, pioglitazone shows beneficial effects on NAFLD rats, but causes body weight gain, which is in accordance with the results in reported clinical studies [42,43]. Moreover, pioglitazone may predispose at-risk patients to exacerbated heart failure [44]. Because of these side effects in addition to other potential risks, efficacy of some of the insulin sensitizers in NAFLD therapy is limited. This study shows that the two regimens for treating NAFLD rats have similar effects. The results also show that SNN rats do not exhibit any body weight gain, which signifies that $\mathrm{SNN}$ is safe and suitable for long-term use.

\section{Conclusions}

The results show that SNN exerts a protective effect on steatosis in HepG2 cells and HC diet-induced NAFLD in rats. This protective effect may be due to attenuation of hepatic insulin resistance.
Competing interests

The authors declare that they have no competing interests.

\section{Authors' contributions}

LZ and GJ designed the study, LZ and JYX carried out experiments. HYS participated in the data analysis, LZ and ZMY interpreted the data, drafted and edited the manuscript, GJ supervised all the experiments. All authors have read and approved the final manuscript.

\section{Acknowledgements}

This study was supported by the National Nature Science Foundation of China (No. 81273727, 81302927), Innovation Program of Shanghai Municipal Education Commission, No.14YZ053.

\section{Author details}

${ }^{1}$ Institute of Digestive Disease, Longhua Hospital, Shanghai University of Traditional Chinese Medicine, Shanghai, China. ${ }^{2}$ Department of Biochemistry, Microbiology \& Immunology, Ottawa Institute of Systems Biology, University of Ottawa, Ottawa, Canada. ${ }^{3}$ E-Institute of Shanghai Municipal Education Commission, Shanghai University of Traditional Chinese Medicine, Shanghai, China.

Received: 12 May 2014 Accepted: 20 August 2014

Published: 27 August 2014

\section{References}

1. Adams LA, Waters OR, Knuiman MW, Elliott RR, Olynyk JK: NAFLD as a risk factor for the development of diabetes and the metabolic syndrome: an eleven-year follow-up study. Am J Gastroenterol 2009, 104(4):861-867.

2. Janczyk W, Socha P: Non-alcoholic fatty liver disease in children. Clin Res Hepatol Gastroenterol 2012, 36(3):297-300.

3. Abdelmalek MF, Diehl AM: Nonalcoholic fatty liver disease as a complication of insulin resistance. Med Clin North Am 2007, 91(6):1125-1149. ix.

4. Marchesini G, Moscatiello S, Di Domizio S, Forlani G: Obesity-associated liver disease. J Clin Endocrinol Metab 2008, 93(11 Suppl 1):S74-S80.

5. Utzschneider KM, Kahn SE: Review: The role of insulin resistance in nonalcoholic fatty liver disease. J Clin Endocrinol Metab 2006, 91(12):4753-4761.

6. Previs SF, Withers DJ, Ren JM, White MF, Shulman Gl: Contrasting effects of IRS-1 versus IRS-2 gene disruption on carbohydrate and lipid metabolism in vivo. J Biol Chem 2000, 275(50):38990-38994.

7. Bowman TA, Ramakrishnan SK, Kaw M, Lee SJ, Patel PR, Golla VK, Bourey RE, Haram PM, Koch LG, Britton SL, Wisloff U, Lee AD, Najjar SM: Caloric restriction reverses hepatic insulin resistance and steatosis in rats with low aerobic capacity. Endocrinology 2010, 151(11):5157-5164.

8. Wang $M$, Sun $S$, Wu T, Zhang $L$, Song H, Hao W, Zheng P, Xing L, Ji G: Inhibition of LXRalpha/SREBP-1c-Mediated Hepatic Steatosis by Jiang-Zhi Granule. Evid Based Complement Alternat Med 2013, 2013:584634.

9. LU Y-L WANGM, ZHANG L, HE Y-Q YANGL, WANG C-H, WANG Z-T JIG: Simultaneous Determination of Six Components in the 'Jiang-Zhi' Granule by UPLC-MS Analysis. Chin J Nat Med 2010, 8(6):449-455.

10. Qi Z, Xue J, Zhang Y, Wang H, Xie M: Osthole ameliorates insulin resistance by increment of adiponectin release in high-fat and high-sucrose-induced Fatty liver rats. Planta Med 2011, 77(3):231-235.

11. Lomba A, Milagro Fl, Garcia-Diaz DF, Marti A, Campion J, Martinez JA: Obesity induced by a pair-fed high fat sucrose diet: methylation and expression pattern of genes related to energy homeostasis. Lipids Health Dis 2010, 9:60.

12. Fernandes-Santos C, Evangelista Carneiro R, De Souza ML, Barbosa Aguila M, Mandarim-de-Lacerda CA: Rosiglitazone aggravates nonalcoholic Fatty pancreatic disease in C57BL/6 mice fed high-fat and high-sucrose diet. Pancreas 2009, 38(3):e80-e86.

13. Song HY, Zhang L, Pan JL, Yang LL, Ji G: Bioactivity of five components of Chinese herbal formula Jiangzhi granules against hepatocellular steatosis. J Integr Med 2013, 11(4):262-268.

14. Lima CR, Vasconcelos CF, Costa-Silva JH, Maranhao CA, Costa J, Batista TM, Carneiro EM, Soares LA, Ferreira F, Wanderley AG: Anti-diabetic activity of extract from Persea americana Mill. leaf via the activation of protein kinase $B(\mathrm{PKB} / \mathrm{Akt})$ in streptozotocin-induced diabetic rats. J Ethnopharmacol 2012, 141(1):517-525. 
15. Villanueva CJ, Monetti M, Shih M, Zhou P, Watkins SM, Bhanot S, Farese RV Jr: Specific role for acyl CoA:Diacylglycerol acyltransferase 1 (Dgat1) in hepatic steatosis due to exogenous fatty acids. Hepatology 2009, 50(2):434-442.

16. Xing $L$, Zhang $L$, Liu T, Hua YQ, Zheng PY, Ji G: Berberine reducing insulin resistance by up-regulating IRS-2 mRNA expression in nonalcoholic fatty liver disease (NAFLD) rat liver. Eur J Pharmacol 2011, 668(3):467-471.

17. Ueki K, Kondo T, Kahn CR: Suppressor of cytokine signaling 1 (SOCS-1) and SOCS-3 cause insulin resistance through inhibition of tyrosine phosphorylation of insulin receptor substrate proteins by discrete mechanisms. Mol Cell Biol 2004, 24(12):5434-5446.

18. Rui L, Yuan M, Frantz D, Shoelson S, White MF: SOCS-1 and SOCS-3 block insulin signaling by ubiquitin-mediated degradation of IRS1 and IRS2. J Biol Chem 2002, 277(44):42394-42398.

19. Considine RV, Nyce MR, Allen LE, Morales LM, Triester S, Serrano J, Colberg J, Lanza-Jacoby S, Caro JF: Protein kinase $C$ is increased in the liver of humans and rats with non-insulin-dependent diabetes mellitus: an alteration not due to hyperglycemia. J Clin Invest 1995, 95(6):2938-2944.

20. Pan J, Wang M, Song H, Wang L, Ji G: The efficacy and safety of traditional chinese medicine (jiang zhi granule) for nonalcoholic Fatty liver: a multicenter, randomized, placebo-controlled study. Evid Based Complement Alternat Med 2013, 2013:965723.

21. Fon Tacer K, Rozman D: Nonalcoholic Fatty liver disease: focus on lipoprotein and lipid deregulation. J Lipids 2011, 2011:783976.

22. Musunuru K: Atherogenic dyslipidemia: cardiovascular risk and dietary intervention. Lipids 2010, 45(10):907-914.

23. Poss J, Custodis F, Werner C, Weingartner O, Bohm M, Laufs U: Cardiovascular disease and dyslipidemia: beyond LDL. Curr Pharm Des 2011, 17(9):861-870

24. Charlton M, Sreekumar R, Rasmussen D, Lindor K, Nair KS: Apolipoprotein synthesis in nonalcoholic steatohepatitis. Hepatology 2002, 35(4):898-904

25. Ota T, Gayet C, Ginsberg HN: Inhibition of apolipoprotein B100 secretion by lipid-induced hepatic endoplasmic reticulum stress in rodents. J Clin Invest 2008, 118(1):316-332.

26. Day CP, James OF: Steatohepatitis: a tale of two "hits"? Gastroenterology 1998 114(4):842-845.

27. Jou J, Choi SS, Diehl AM: Mechanisms of disease progression in nonalcoholic fatty liver disease. Semin Liver Dis 2008, 28(4):370-379.

28. Gayoso-Diz P, Otero-Gonzalez A, Rodriguez-Alvarez MX, Gude F, Cadarso-Suarez C, Garcia F, De Francisco A: Insulin resistance index (HOMA-IR) levels in a general adult population: curves percentile by gender and age. The EPIRCE study. Diabetes Res Clin Pract 2011, 94(1):146-155.

29. Mari A, Pacini G, Murphy E, Ludvik B, Nolan JJ: A model-based method for assessing insulin sensitivity from the oral glucose tolerance test. Diabetes Care 2001, 24(3):539-548

30. Ben Djoudi Ouadda A, Levy E, Ziv E, Lalonde G, Sane AT, Delvin E, Elchebly M: Increased hepatic lipogenesis in insulin resistance and Type 2 diabetes is associated with AMPK signalling pathway up-regulation in Psammomys obesus. Biosci Rep 2009, 29(5):283-292.

31. Zheng D, lonut V, Mooradian V, Stefanovski D, Bergman RN: Exenatide sensitizes insulin-mediated whole-body glucose disposal and promotes uptake of exogenous glucose by the liver. Diabetes 2009, 58(2):352-359.

32. Biddinger SB, Hernandez-Ono A, Rask-Madsen C, Haas JT, Aleman JO, Suzuki R, Scapa EF, Agarwal C, Carey MC, Stephanopoulos G, Cohen DE, King GL, Ginsberg $\mathrm{HN}$, Kahn CR: Hepatic insulin resistance is sufficient to produce dyslipidemia and susceptibility to atherosclerosis. Cell Metab 2008, 7(2):125-134.

33. Farese RV Jr, Zechner R, Newgard CB, Walther TC: The Problem of Establishing Relationships between Hepatic Steatosis and Hepatic Insulin Resistance. Cell Metab 2012, 15(5):570-573.

34. Bugianesi E, McCullough AJ, Marchesini G: Insulin resistance: a metabolic pathway to chronic liver disease. Hepatology 2005, 42(5):987-1000.

35. Zhang Y, Lee FY, Barrera G, Lee H, Vales C, Gonzalez FJ, Willson TM, Edwards PA: Activation of the nuclear receptor FXR improves hyperglycemia and hyperlipidemia in diabetic mice. Proc Natl Acad Sci U S A 2006, 103(4):1006-1011.

36. Ros S, Garcia-Rocha M, Calbo J, Guinovart JJ: Restoration of hepatic glycogen deposition reduces hyperglycaemia, hyperphagia and gluconeogenic enzymes in a streptozotocin-induced model of diabetes in rats. Diabetologia 2011, 54(10):2639-2648.

37. Ha Do T, Trung TN, Hien TT, Dao TT, Yim N, Ngoc TM, Oh WK, Bae K: Selected compounds derived from Moutan Cortex stimulated glucose uptake and glycogen synthesis via AMPK activation in human HepG2 cells. J Ethnopharmacol 2010, 131(2):417-424.

38. Schmitz-Peiffer C, Biden TJ: Protein kinase $C$ function in muscle, liver, and beta-cells and its therapeutic implications for type 2 diabetes.

Diabetes 2008, 57(7):1774-1783

39. Hribal ML, D'Alfonso R, Giovannone B, Lauro D, Liu YY, Borboni P, Federici M, Lauro R, Sesti G: The sulfonylurea glimepiride regulates intracellular routing of the insulin-receptor complexes through their interaction with specific protein kinase C isoforms. Mol Pharmacol 2001, 59(2):322-330.

40. McCowen KC, Fajtova VT: Pioglitazone for diabetes prevention. N Engl J Med 2011, 365(2):182-183. author reply 183-184.

41. Aithal GP, Thomas JA, Kaye PV, Lawson A, Ryder SD, Spendlove I, Austin AS, Freeman JG, Morgan L, Webber J: Randomized, placebo-controlled trial of pioglitazone in nondiabetic subjects with nonalcoholic steatohepatitis. Gastroenterology 2008, 135(4):1176-1184.

42. Kusunoki M, Tsutsumi K, Sato D, Nakamura A, Habu S, Mori Y, Morishita M, Yonemoto T, Miyata T, Nakaya Y, Nakamura T: Pioglitazone-induced body weight gain is prevented by combined administration with the lipoprotein lipase activator NO-1886. Eur J Pharmacol 2011, 668(3):486-491.

43. Sanyal AJ, Chalasani N, Kowdley KV, McCullough A, Diehl AM, Bass NM, Neuschwander-Tetri BA, Lavine JE, Tonascia J, Unalp A, Van Natta M, Clark J, Brunt EM, Kleiner DE, Hoofnagle JH, Robuck PR, Nash CRN: Pioglitazone,

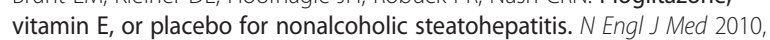
362(18):1675-1685.

44. Lincoff AM, Wolski K, Nicholls SJ, Nissen SE: Pioglitazone and risk of cardiovascular events in patients with type 2 diabetes mellitus: a meta-analysis of randomized trials. JAMA 2007, 298(10):1180-1188.

doi:10.1186/s12967-014-0236-8

Cite this article as: Zhang et al:: Extracts from Salvia-Nelumbinis naturalis alleviate hepatosteatosis via improving hepatic insulin sensitivity. Journal of Translational Medicine 2014 12:236.

\section{Submit your next manuscript to BioMed Central and take full advantage of:}

- Convenient online submission

- Thorough peer review

- No space constraints or color figure charges

- Immediate publication on acceptance

- Inclusion in PubMed, CAS, Scopus and Google Scholar

- Research which is freely available for redistribution
C) BioMed Central 